\title{
Neuropsychiatric Manifestations of Celiac Disease in Young Child - Case Report
}

\author{
Mircea Margescu ${ }^{1,2, *}$, Camelia Carmen Margescu ${ }^{1}$, Rodica Elena Pantis ${ }^{3}$, MadalinaIonela Chiriac ${ }^{4}$, Rodica Cornean $^{1,2}$ \\ ${ }^{1}$ II- nd Pediatric Department of Clinical Pediatrics Emergency Hospital Cluj-Napoca, Romania \\ ${ }^{2}$ Phd "Iuliu Hatieganu” University of Medicine and Pharmacy, Cluj-Napoca, Romania \\ ${ }^{3}$ Pediatric Psychiatry Department of Clinical Pediatrics Emergency Hospital Cluj-Napoca, Romania \\ ${ }^{4}$ Pediatric Surgery Department of Clinical Pediatrics Emergency Hospital Cluj-Napoca, Romania \\ *Corresponding author: mirceammv@yahoo.com
}

Received April 06, 2015; Revised April 29, 2015; Accepted May 04, 2015

\begin{abstract}
Neurological and psychiatric symptoms represent an atypical manifestation of celiac disease (CD) occurring before the typical gastrointestinal events (weight loss, diarrhea, steatorrhoeic stools). Some studies suggest that a gluten-free diet is effective in treating psychiatric and neurological complications associated with CD. We report the case of 1 year and 10 months female child presented in II-nd Pediatrics Clinic of Clinical Pediatrics Emergency Hospital Cluj-Napoca with important neuropsychiatric manifestations (psychomotor tension, sobbing spasms, affective lability, separation anxiety and social anxiety, non-organic disorders sleeping) and typical manifestations of CD (loss of appetite, weight loss). The family history (paternal grandfather diagnose with intestinal lymphoma, mother suffering of type I diabetes and autoimmune thyroiditis - AT) relive the association with other autoimmune diseases and possible complications of CD. Complete remission of all clinical manifestations under the gluten confirms that symptoms were in the context of CD.
\end{abstract}

Keywords: neuro-psychiatric manifestations, child, celiac disease, autoimmunity

Cite This Article: Mircea Margescu, Camelia Carmen Margescu, Rodica Elena Pantis, Madalina Ionela Chiriac, and Rodica Cornean, "Neuropsychiatric Manifestations of Celiac Disease in Young Child - Case Report.” International Journal of Celiac Disease, vol. 3, no. 2 (2015): 69-71. doi: 10.12691/ijcd-3-2-4.

\section{Introduction}

Celiac disease (CD) is an autoimmune disorder that occurs in genetically predisposed individuals trigged by gluten. Neurological and psychiatric symptoms represent an atypical manifestation of $\mathrm{CD}$ that can occure before the typical gastrointestinal events (weight loss, diarrhea, steatorrhoeic stools) [1,2]. CD has been associated with neurological disorders (cognitive function deterioration, cerebral ataxia, brain atrophy, peripheral neuropathy, epilepsy) and psychiatric disorders such as apathy, anxiety and irritability are usually the most frequent symptoms described in literature [3,4]. Some studies suggest that gluten-free diet is effective in treating depression, anxiety and associated neurological complications of CD [5]. The authors report the case of a little child with neuropsychological symptoms which started after the introduction of gluten in diet and fast remission of symptoms after its exclusion from the diet.

\section{Case Report}

1 year and 10 months female child is hospitalized in our clinic for important psychomotor tension, sleep disturbances, seizures, affective lability, anorexia and then slowly lose weight. From her family history we notice that her paternal grandfather died of intestinal lymphoma and her mother was diagnosed with Type I diabetes and autoimmune thyroiditis (AT). From her personal physiological history we also acknowledged that the girl comes from a pathological pregnancy (diabetic mother), born at term with $\mathrm{W}=5100 \mathrm{~g}, \mathrm{H}=53 \mathrm{~cm}$, APGAR score $=9$, was fed with breast milk for one month, then with formula milk powder, the diversifications started at age of 5 months. The gluten was introduced into the diet at age 5 and half months. From previous medical history we observe repeated upper airway respiratory infections. The clinical manifestations previously presented, insidiously started around the age of 16 months, when the mother noticed loss of appetite, weight slowly upward curve, then stationary and especially the appearance of psychomotor tension accompanied by sleep disorders. In the coming months the child increasingly accused irritable moods, affective crises associated with separation anxiety (against them) and social (against foreigners), sometimes with extreme psychomotor tension, sob, reducing communication with her parents. Moreover, the appetite decreases to anorexia with weight loss occurrence, whilst appearance and consistency of stools was normal.

Physical examination at admission revealed altered general state: irritability, hyperkinesias, anxiety about the examiner, pale face, poor nutritional status (actual Weight-aW $=11,500 \mathrm{~g}, \mathrm{aW} / \mathrm{birthW}=0.84$, Height $=84 \mathrm{~cm}$, 
Body mass index $=16,42 \mathrm{~kg} / \mathrm{m}^{2}$ ) lower subcutaneous tissue at the abdomen and thorax, abdomen distended without hepatosplenomegaly, painless on palpation.

Neurological and psychiatric examination was performed and concluding: hyperkinetic syndrome, affective lability, separation anxiety, social anxiety, nonorganic sleep disorders, eating disorders and sobbing spasms. Laboratory examinations revealed mild iron deficiency anemia, normal values of biochemical and immunological investigations (with normal serum immunoglobulin), anti-transglutaminase type A antibodies $($ ATTG) $=148 \mathrm{U} / \mathrm{mL}$ (normal value $>10 \mathrm{U} / \mathrm{mL}$ ), antiendomisium type A antibodies was positive.

Duodenal biopsy was performed; revealing intestinal villous atrophy (observed exocytosis Figure 1a) and intestinal crypts atrophy, moderate fibrosis and inflammation (Figure 1a and 1b).

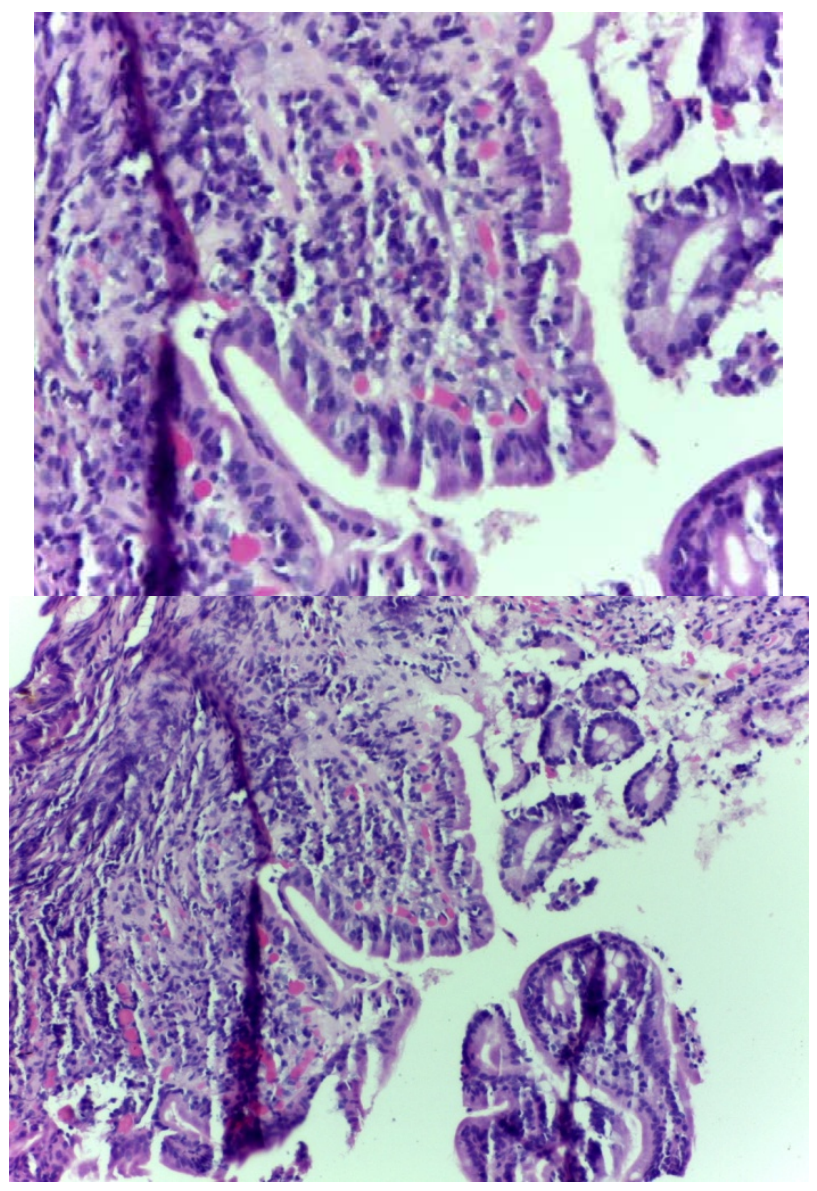

Figure 1. (a $x$ 100, b $-x$ 200) revealed intestinal villous atrophy (observed exocytosis Figure 1a) and intestinal crypts atrophy, moderate fibrosis and inflammation.

The treatment established in our case was a gluten-free diet and lactose free formula milk. Clinical outcome was favorable with complete remission of clinical manifestations, the return of appetite, weight gain upward (1000g in 2 months). The specific antibodies were negative at 2 month evaluation and associated with gluten free diet confirmed the diagnosis of CD.

\section{Discussions}

The case presented in this paper is important by its peculiarities; neuropsychiatric symptoms represent an atypical manifestation of CD, which occurred in an infant with significant family history. In our case the psychiatric manifestations were highlighted by psychomotor tension, sobbing spasm seizures, hyperkinetic syndrome, anxiety and sleep disorders. These events have led the parents to a neurological evaluation and then to psychiatric examination. The worsening general status (anorexia, weight loss and then stagnation) raised the suspicion of $\mathrm{CD}$ in the absence of typical symptoms (chronic diarrhea, steatorrhea stools).

The neuropsychological manifestations associated with CD include brain ataxia [6], peripheral neuropathy [7], depression and epilepsy with cerebral calcifications [8]. CD association with autism remains controversial; the results of different studies are contradictory [4]. The pathogenesis mechanism of mental disorders related to CD is unknown, but disruptions affecting bioavailability of tryptophan and central serotonergic function may play an important role [2].

In our case, the diagnosis of CD was difficult considering the young age of the child and atypical manifestations of CD (the child accused irritability, emotional and behavioral disorders).

The histology of duodenal biopsies was not characteristic of a particular entity, histological criteria for CD was not clear but considering the positive serology was decided to initiate the gluten-free diet. Completed and fast disappearance of the clinical symptoms after setting gluten-free diet is clear evidence that neuropsychiatric manifestations were secondary to $\mathrm{CD}$ and there were not associated another pathology.

Pynnönen et. al. have analyzed the possible effects of psychiatric symptoms on a gluten-free diet, hormonal status and elevated serum amino acid levels in patients with CD. They suggest that serotonergic dysfunction caused by vulnerability disorders occurring in tryptophan availability at people with untreated CD can caused behavior disorders and depression. Adolescents with CD showed improvement in psychiatric symptoms after starting a gluten-free diet, altogether with a significant decrease level of specific antibody for CD and prolactin level, and a significant increase of L-tyrosine serum level $[9,10]$.

Neurological manifestations are estimated at $10 \%$ of patients with $\mathrm{CD}$ and the level of anti-neuronal antibody (AN) in the central nervous system (CNS) and the enteric nervous system (SNE) are found in a significant proportion. Caio et. al reported a case-control study in which they assessed the NA and correlation with neurological and intestinal manifestations in 106 patients with CD by indirect immunofluorescence. They concluded that in $\mathrm{CD}$, the AN antibodies positivity in CNS may be considered a marker of neurologic manifestations. A high level of AN antibody found in NES associated with severe constipation. The fact that AN antibodies were identified both CNS and NES suggest the common immunemediated pathway [11].

Family history is another important feature of the case, paternal grandfather dying due to intestinal lymphoma, representing a major complication that occurs in the natural history of CD [12]. The mother of child diagnosed with Type I diabetes and AT; there are two autoimmune disorders often associated with CD. In this context, 
specific antibodies for CD were investigated at mother but were negative.

According to the latest guidelines of the American College of Clinical Gastroenterology for diagnosis and management of celiac disease, this condition is more common in patients with type I diabetes than in the general population, ranging between $3 \%$ and $10 \%$. In children with type I diabetes, screening for CD is recommended annually or every two years. Moreover, duodenal biopsy is recommended in patients with type I diabetes that performs an upper gastrointestinal endoscopy to exclude CD, especially if they had not previously performed a duodenal biopsy. Thyroid disorders are also twice common in people with CD than in the general population [13]. In our case the child will need to be monitored for a possible association with diabetes mellitus according to statistics 5-10\% [1] and AT. AT is associated with a frequency of $5-7 \%$ in patients with CD [2,14,15].

The clinical outcome under gluten free diet was favorable with disappearance of symptoms and specific serology negative, confirming the diagnosis of CD.

\section{Conclusions}

CD with neuropsychiatric manifestations is rare especially and even more in young children. Family history, typical manifestations of CD (decreased appetite, weight loss) associated with atypical symptom (irritability progressive) guided the diagnosis, confirmed by serological examination and gluten-free diet. Remission of all clinical manifestations under gluten-free diet is the best proof in this case of a diagnosis for CD.

\section{References}

[1] Husby S, Koletzko S, Korponay-Szabo' IR et al. European Society for Pediatric Gastroenterology, Hepatology, and Nutrition guidelines for the diagnosis of coeliac disease. J Pediatr Gastroenterol Nutr. 2012; 54: 136-160.
[2] Freeman HJ, Gujral N and Thomson ABR. Celiac Disease assays in A. B. R. Thomson and E. A. Shaffer, Editors. First Principles of Gastroenterology and Hepatology. Canada: Canadian Academic Publishers Ltd, 2012, Chapter 8: Celiac Disease, pg. 229-256.

[3] Urban-Kowalczyk M, Śmigielski J, Gmitrowicz A. Neuropsychiatric symptoms and celiac disease. Neuropsychiatr Dis Treat 2014; 10: 1961-1964.

[4] Jackson J. R, Eaton W. W, Cascella N. G, Fasano A, Kelly D. L. Neurologic and Psychiatric Manifestations of Celiac Disease and Gluten Sensitivity. Psychiatr Q. 2012; 83: 91-102.

[5] Pynnönen PA, Isometsä ET, Verkasalo MA, et al. Gluten-free diet may alleviate depression and behavioural symptoms in adolescents with coeliac disease: a prospective follow-up case-series study. BMC Psychiatry. 2005; 5: 14.

[6] Burk K, Bosch S., Muller C. A. et al. Sporadic cerebellar ataxia associated with gluten sensitivity. Brain 2001; 124: 1013-1019.

[7] Chin RL, Sander HW, Brannagan TH, Green PH, Hays AP, Alaedini A, Latov N. Celiac neuropathy. Neurology. 2003; 60: 1581.

[8] Garud S, Leffler D, Dennis M, Edwards-George J, Saryan D, Sheth S. Interaction between psychiatric and autoimmune disorders in celiac disease patients in the Northeastern United States. Aliment Pharmacol Ther. 2009; 29: 898-905.

[9] Pynnönen P. A, Isometsä E. T, Verkasalo M. A, Kähkönen S. A, Sipilä I, Savilahti E and Aalberg V. A. Gluten-free diet may alleviate depressive and behavioural symptoms in adolescents with coeliac disease: a prospective follow-up case-series study. BMC Psychiatry 2005; 5: 14.

[10] Pynnönen P, Isometsä E, Aronen E, Verkasalo M, Savilahti E, Aalberg V: Mental disorders in adolescents with celiac disease. Psychosomatics 2004; 45: 325-335.

[11] Caio G, De Giorgio R, Venturi A, Giancola F, Latorre R, Boschetti E, Serra M, Ruggeri E, Volta U. Clinical and immunological relevance of anti-neuronal antibodies in celiac disease with neurological manifestations. Gastroenterol Hepatol Bed Bench 2015; 8: 146-152.

[12] Ahin S, Oban C, Ensari A, Idilman R, Köklü S, Yolcu OF, Ormeci N. Intestinal B Cell Lymphoma Associated with Chronic Hepatitis C and Celiac Disease. Dig Dis Sci 2005; 50: 2359-2361.

[13] Rubio-Tapia A, Hill ID, Kelly CP, Calderwood AH, Murray JA. American College of Gastroenterology. ACG clinical guidelines: diagnosis and management of celiac disease. Am J Gastroenterol. 2013; 108: 656-677.

[14] Aggarwal S, Lebwohl B and Green PHR. Screening for celiac disease in average-risk and high-risk populations. Therap Adv Gastroenterol 2012; 5: 37-47.

[15] Stanescu A. M. Boala celiaca la copil si adolescent. Corelatii anatomo-clinice. Teza de Doctorat. Bucuresti, 2006. 